\title{
The thermal expansion of erbium over a wide temperature range
}

\author{
Yurii Kozlovskii ${ }^{*}$ \\ Kutateladze Institute of Thermophysics SB RAS, 630090 Novosibirsk, Russia
}

\begin{abstract}
A dilatometric study results on the linear thermal expansion coefficient (LTEC) of polycrystalline erbium are presented. The experimental data in the temperature range of $100-800 \mathrm{~K}$ are obtained. The measurements were made with an error 3\%. Approximate dependencies which allow calculating the reference tables of thermal properties for the entire measurement interval are defined.
\end{abstract}

\section{Introduction}

Rare earth metals (REM) are widely used in many fields of science and technology and at the creation of materials with unique properties [1-3]. One of the determining factors at the development and operation of parts and optimization of the processes of the synthesis of alloys and compounds of the REM are the thermophysical properties including thermal expansion. However, data on the linear thermal expansion coefficient (LTEC) of erbium were obtained in the last century and differ [4-7]. Therefore, existing results require verification and clarification. In addition, modern experimental equipment allows carry out measurements over a wide temperature range with high accuracy [8]. Thus, the purpose of this work is to study the thermal expansion of polycrystalline erbium over a wide temperature range.

\section{Material, method, and experimental equipment}

Distilled erbium, previously melted in a tantalum crucible, with a purity of 99.83 mass. $\%$ was used to prepare the samples. The main impurities at material: $\mathrm{Fe}-0.01 \%$; $\mathrm{Ca}-$ $0.01 \%$; $\mathrm{Cu}-0.03 \%$; refractory metals-0.02\%; others REM $-0.1 \%$. The polycrystalline specimens were the shape of cylinders $25 \mathrm{~mm}$ long and $6 \mathrm{~mm}$ diameter with plane base. They were annealed in vacuum during 4 hours at a temperature of $1273 \mathrm{~K}$ before main experiments.

The thermal expansion of erbium was investigated using a DIL-402C horizontal dilatometer [9] with holder and pushrod made of fused silica or sintered alumina. The sample was mounted on a stand made of holder material and was clamped between the holder and pushrod with a load of $45 \mathrm{cN}$ which was held constant during the experiment. The elongation was measured by an inductive linear variable displacement transducer

*Corresponding author: kozlovskii.yurii@gmail.com 
(LVDT) with a resolution better than $1 \mathrm{~nm}$. The temperature was measured with type $\mathrm{E}$ (for low temperatures) and type $S$ (for high temperatures) thermocouples. The hot junction of the thermocouple was located in direct proximity to the lateral surface of the sample. The measurements were carried out in the temperature range 100-800 K during heating and cooling at a rate of $2 \mathrm{~K} / \mathrm{min}$ and $30 \mathrm{~min}$ isothermal exposure at maximum and minimum temperatures. The experiments were carried out in helium (99.995 vol. \% pure) which was additionally purified by special device of cleaning and drying of inert gases - EPISHURA 11 SL [10]. A detailed description of the experimental technique was given in the previous paper [8].

The instrumental error was determined on standard samples of fused silica (for low temperatures) and sintered alumina (for high temperatures). The results were reproduced within $0.2 \mu \mathrm{m}$. The systematic error of the LTEC was determined in experiments with samples of high-purity aluminum, copper (99.99 mass \% pure) and platinum (99.93 mass \% pure). The results showed that the difference between the data obtained in the present work and the reference data of the LTEC [11,12] doesn't exceed $(1.5-2) \times 10^{-7} \mathrm{~K}^{-1}$ (or $3 \%$ ) and on average is $8 \times 10^{-8} \mathrm{~K}^{-1}$. All experiments were under conditions identical to those of the main experiments with erbium.

Measurement results was given as the temperature dependence of the relative elongation $\varepsilon$ of the sample under heating or cooling:

$$
\varepsilon(T)=\left(L(T)-L_{293}\right) / L_{293}
$$

where $L_{293}$ and $L(T)$ are the sample length at $293.15 \mathrm{~K}$ and at $T$, respectively. The LTEC $\alpha$, and the true LTEC $\alpha^{*}$ are determined as follows:

$$
\begin{aligned}
& \alpha(T)=\frac{1}{L_{293}}\left(\frac{\partial L}{\partial T}\right)_{p}=\left(\frac{\partial \varepsilon}{\partial T}\right)_{p} \\
& \alpha^{*}(T)=\frac{1}{L}\left(\frac{\partial L}{\partial T}\right)_{p}=\frac{\alpha(T)}{1+\varepsilon(T)},
\end{aligned}
$$

where $p$ is the pressure.

The temperature dependence of the LTEC was found by direct numerical differentiation of the raw experimental data on relative elongation:

$$
\alpha\left(T_{i}\right)=\frac{1}{2}\left(\frac{\varepsilon_{i+1}-\varepsilon_{i}}{T_{i+1}-T_{i}}+\frac{\varepsilon_{i}-\varepsilon_{i-1}}{T_{i}-T_{i-1}}\right)
$$

where $\varepsilon_{i}=\varepsilon\left(T_{i}\right)$ is the relative elongation of the sample at the temperature $T_{i}$. Such approach makes it possible to obtain directly the temperature dependence of the LTEC, without differentiating the approximation equation for the relative elongation. The last one introduces an additional error into the $\alpha$ associated with the ambiguity in the choice of the fitting equation for $\varepsilon(T)$.

\section{Results and Discussion}

The experiments were carried out in two stages: at low and high temperatures. The obtained data were «sewed together» in overlapping regions. The temperatures of the boundaries of the regions were determined from the condition of equality of the LTEC. 


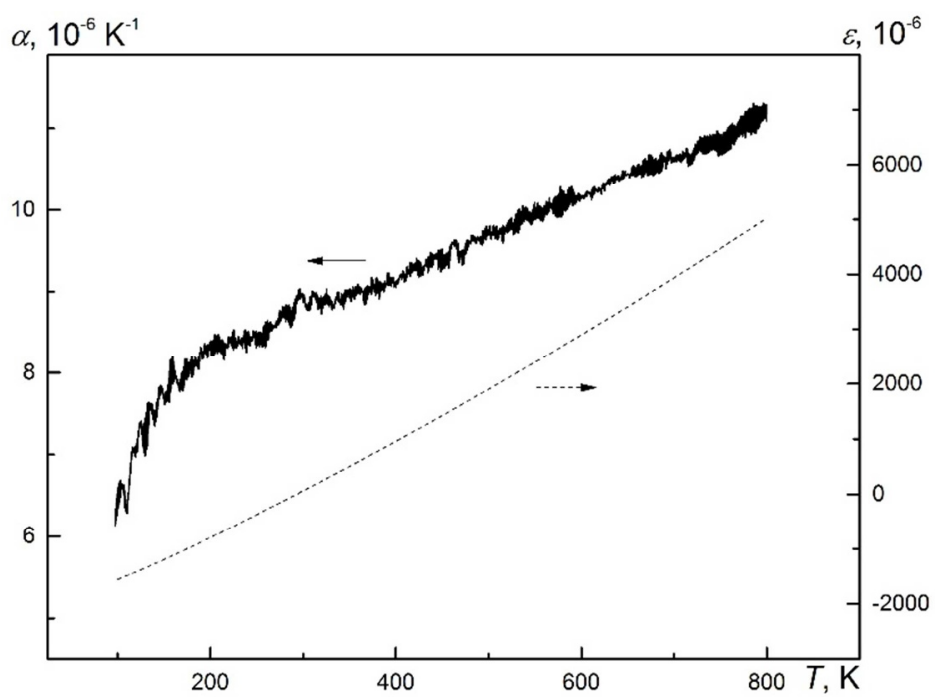

Fig. 1. Typical measurement results of the thermal expansion and the relative elongation of polycrystalline erbium.

Figure 1 shows the thermal expansion results of erbium. The raw data were fitted by the least-squares method with equations of the form:

$$
\alpha(T)=\sum_{i=0}^{k} A_{i} t^{i},
$$

where $t=T-273.15 \mathrm{~K}$. Coefficients of the equation (4) are presented in table 1 .

Table 1. Coefficients of the equation (4).

\begin{tabular}{cccccc}
\hline Region & $T, \mathrm{~K}$ & $A_{0}, \mathrm{~K}^{-1}$ & $A_{1}, \mathrm{~K}^{-1}$ & $A_{2}, \mathrm{~K}^{-1}$ & $A_{3}, \mathrm{~K}^{-1}$ \\
\hline 1 & $100-282$ & 1.1614 & $77.296 \times 10^{-3}$ & $-2.7834 \times 10^{-4}$ & $3.4924 \times 10^{-7}$ \\
2 & $282-779$ & 7.301 & $4.778 \times 10^{-3}$ & - & - \\
3 & $779-800$ & -43.1119 & $24.4509 \times 10^{-2}$ & $36.9512 \times 10^{-5}$ & $18.595 \times 10^{-8}$ \\
\hline
\end{tabular}

Figure 2 shows a comparison of the obtained results on the LTEC of erbium with the known data from the other sources. As you can see, the data strongly diverge. The maximum difference with the reference dependence $\alpha(T)$ [4] is observed at low temperatures and reaches $21 \%$. The values in work [3] are also higher than those obtained by us. However, the difference does not exceed $9 \%$. In the region above room temperature, erbium is better investigated. Good agreement is observed with work [7]. The results coincide within the limits of the measurement error of our work, but exceed the confidence limits (95\%) of the random error of approximation. In addition, the method used in [7] has a lower accuracy. Perhaps, discrepancy between the experimental data of various authors is a consequence of the different purity of the samples being studied and the specifics of their preparation. Since it is known that non-metallic impurities affect the coefficient of the thermal expansion [13]. 


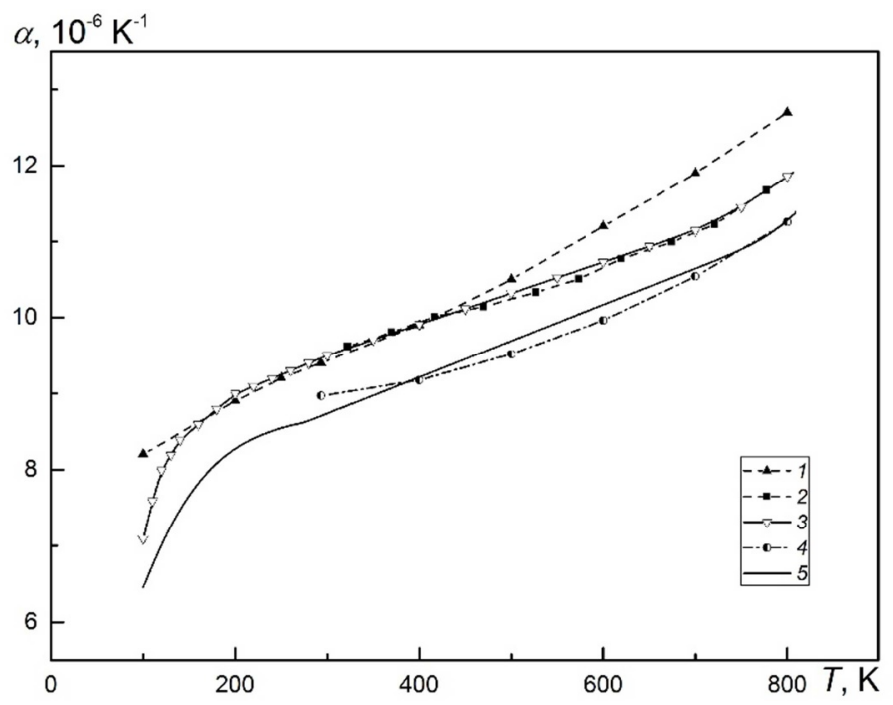

Fig. 2. Recommended data for the thermal expansion of erbium $1-[4] ; 2-[5] ; 3-[6] ; 4-[7] ; 5$ - this work.

\section{Conclusion}

The experimental data on the linear thermal expansion coefficient of polycrystalline erbium are obtained. The results obtained have lower values than the data of most known works. It is established that the purity of the initial material and the preliminary treatment of the samples affect the LTEC of erbium. The paper presents recommended approximation equations applicable over a wide temperature range. This allows creating detailed data tables of the thermal expansion and the relative elongation of erbium.

\section{References}

1. I. Ishikawa, A. Aoki, A.A. Takasaki, J. Int. Acad. Periodontol. 10(1), 22 (2008)

2. L.-N. Sun, H.-J. Zhang, L.-S. Fu, F.-Y. Liu, et al., Adv. Funct. Mater. 15, 1041 (2005)

3. K. Seneschal, F. Smektala, B. Bureau, M. Le Floch, et al., Mater. Res. Bul. 40, 1433 (2005)

4. Y.S. Touloukian, R.K. Kirby, R.E. Taylor, P.D. Desai, Thermal expansion. Metallic elements and alloys (Thermophys. Prop. Matter., IFI / Plenum, 1975)

5. F. Barson, S. Legvold, F.H. Spedding, Phys. Rev. 105, 418 (1957)

6. S.I. Novikova, Thermal Expansion of Solids (Nauka, 1974)

7. S.V. Stankus, P.V. Tyagel'sky, A.V. Baginskii, K.M. Lyapunov, High Temp. 27/28, 485 (1995)

8. R.N. Abdullaev, Yu.M. Kozlovskii, R.A. Khairulin, S.V. Stankus, Int. J. Thermophys. 36, 603 (2015)

9. https://www.netzsch-thermal-analysis.com/en/products-solutions/dilatometer

10. http://granat-e.ru/stend_epishur-a_sl

11. F.R. Kroeger, C.A. Swenson, J. Appl. Phys. 48, 853 (1977)

12. R.K. Kirby, Int. J. Thermophys. 12, 679 (1991)

13. B.J. Beaudry and F.H. Spedding, Met. Trans. 6B, 419 (1975) 